\title{
Development of a Wheeled and Wall-pressing Type In-Pipe Robot for Water Pipelines Cleaning and its Traveling Capability
}

\author{
Guanhua FENG*,**, Wenhao LI*,**, Zhigang LI***, Zhen HE*** \\ *Institute of Mechanics, Chinese Academy of Sciences, Beijing 100190, P. R. China, E-mail: liwenhao@imech.ac.cn \\ **School of Engineering Sciences, University of Chinese Academy of Sciences, Beijing 100049, P. R.China, \\ E-mail: liwenhao@imech.ac.cn (Corresponding author) \\ ***State Key Laboratory of Robotics, Shenyang Institute of Automation, Chinese Academy of Sciences, Shenyang 110016, \\ P. R. China, E-mail: lzg@sia.cn
}

cross $^{\text {ref }}$ http://dx.doi.org/10.5755/j01.mech.26.2.18783

\section{Introduction}

Recently, pipelines, being widely applied in many fields, are the safest, environmentally friendly, energy-efficient, and economic way to transport goods or material, such as gas, oil and water etc. [1-3]. However, with the long-term transportation, corrosion of transported medium and environmental effects, there will be sediment and it is possible to block the pipe when the sediment accumulates to a certain level in the time, causing accidents and heavy loss [4].

Certainly, water pipelines are useful for transporting drinking water, improving the quality of people's life greatly. New pipelines need to be thoroughly cleaned to transport drinking water, whereas the serious corrosion and sediment appear in old pipelines for long-term operation, not only reducing efficiency of water supplies because of the de-crease of cross sectional area, also affecting water quality and threatening people's health and safety [5]. So these problems motivated the development of in-pipe mechanism for pipelines cleaning [6-7].

\subsection{Development of IPCR}

Until now, many in-pipe robots have been developed by researchers, but most are used for maintenance, inspection in conventional pipeline and "cursory" cleaning inside ventilating or central air-conditioning pipelines [8-13]. There is almost no in-pipe cleaning robot with high pressure water jet for cleaning large diameter pipelines, most of which are unpowered and towed-streamer in-pipe cleaning bracket drawn by auxiliary devices outside pipelines, as shown in Fig. 1, a [14]. In-pipe jet cleaning robot designed by Stoneage company in America, as shown in Fig. 1, b, is the only in-pipe caterpillar type robot equipped with high pressure water jet at the level of application, but it still has problems:

1. Poor capability to suit multi-radius pipelines.

2. The robot cannot travel in the curved pipelines.

3. The cleaning effect is unsatisfactory.

4. The energy supply is unreliable so that the robot cannot achieve long distance cleaning task [14].

Considering the problems above and to finish the in-pipe cleaning task well, we proposed a novel IPCR with high pressure water jet and must consider some key factors in our design:

1. Powerful with traction force.

2. Wide application range of pipe diameters, especially the large diameter.
3. Mobility in curved pipelines.

4. Good cleaning effect and high energy efficiency.

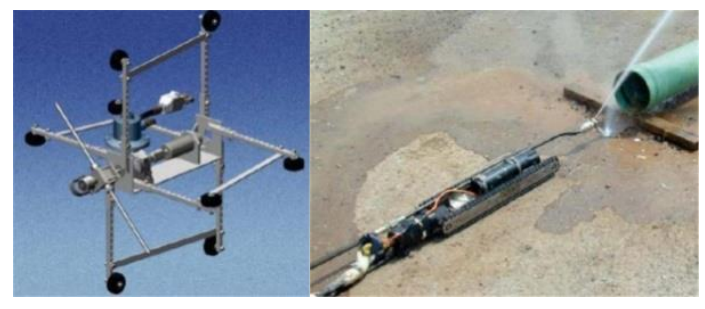

a

b

Fig. 1 Pipelines cleaning: $\mathrm{a}$ - bracket with no power, $\mathrm{b}$-inpipe jet cleaning robot of Stoneage company

\subsection{Integration of mechanisms}

In this paper, the proposed IPCR for water pipelines cleaning was introduced, and it was applied to the water pipelines whose diameter is range from 1200 to $1500 \mathrm{~mm}$ and filled or half-filled with water. And its objective distance of cleaning operation should reach $1500 \mathrm{~m}$ and operation speed is adjustable. Fig. 2 is the cleaning system of water pipelines we proposed, including cleaning robot (pulling cables and water pipe), winch of cables and water pipe, control system, power system, etc., where winch, control system and power system are outside the water pipelines.

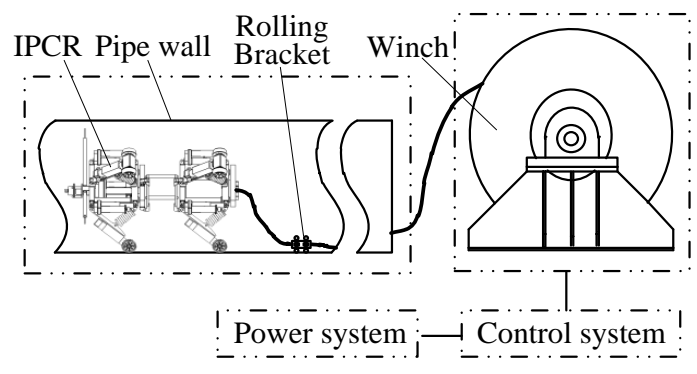

Fig. 2 Cleaning system of water pipelines

Now, the mechanisms of in-pipe robots developed by re-searchers can be classified into eight types [15], and the IPCR with high pressure water jet was designed in this paper by combining two mechanism types, wheeled and wall-pressing type, as shown in Fig. 3. Some basic parameters of the proposed IPCR are listed in Table 1.

As is shown in Fig. 3, a, the main body of IPCR consists of three main parts: front frame, middle frame and rear frame, and it is detachable and convenient for IPCR to 
enter the initial position of water pipe. The front frame has the same mechanisms of wheeled and wall-pressing type with the rear frame. Front frame is designed for carrying water pipe, rotation arm, gear box and two motor shells of rotation arm, whereas rear frame for supporting electronic cabin, each end of which is embedded with twelve connectors with sixteen pins, respectively.

As shown in Fig. 3, front/rear sliding frame is driven by three screw nuts inside push rod shell. Front/rear sliding frame fitted with three sliding bearing bushes can move forward/backward along three pull rods fitted with linear bearing, which it will produce force on swing rod of driving wheels by compressing the spring between swing rod and front/rear sliding frame. By this way, swing amplitude of swing rod can be changed synchronously with the change of diameters, improving the pipelines adaptability and obstacle-overcoming capability of the IPCR; besides, displacement sensors (i.e. reed switch sensors) are fixed on the springs and front/rear sliding frame, measuring the normal force developed between the driving wheels and the wa- ter pipelines. The normal force must be large enough to produce traction force for moving forward further, reaching the objective distance of cleaning operation. To ensure the sufficient traction force of the IPCR, six groups of wheels are all installed with driving motors, and six motors can realize the power balance through control algorithm.

Table 1

Parameters of the IPCR

\begin{tabular}{|c|c|}
\hline Parameters & Value \\
\hline Length, mm & 1700 \\
\hline Mass in the air, kg & 450 \\
\hline Number of driving wheels, group & 6 \\
\hline Number of springs & 6 \\
\hline Stiffness of springs, N/mm & 101 \\
\hline Number of motors & 14 \\
\hline Application of diameter, m & $1.2-1.4$ \\
\hline Length of cables and water pipe, $\mathrm{m}$ & 1600 \\
\hline Operation distance, $\mathrm{m}$ & 1500 \\
\hline Operation speed, $\mathrm{m} / \mathrm{min}$ & $1-5$ \\
\hline Speed of rotation arm, rpm & $10-30$ \\
\hline
\end{tabular}

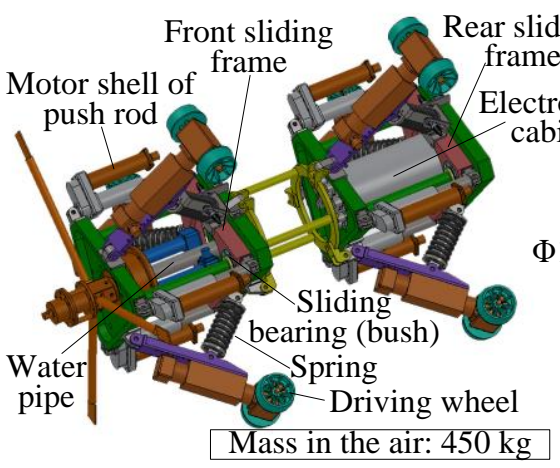

a

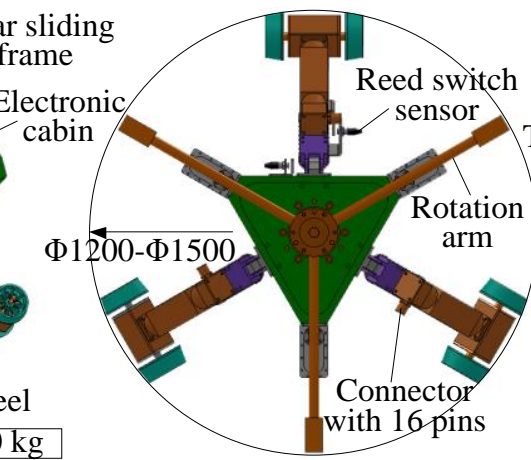

b

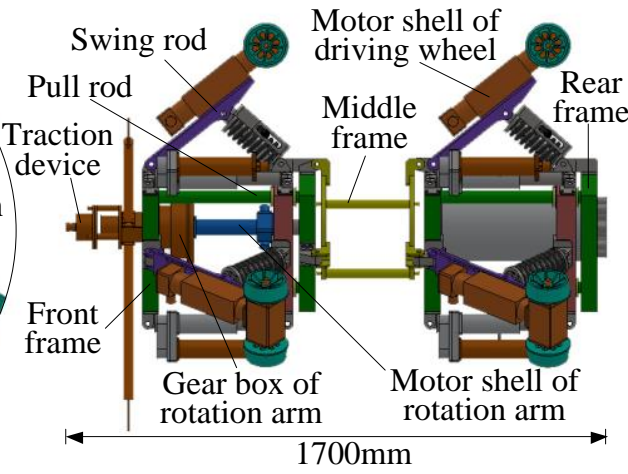

c

Fig. 3 Mechanism of IPCR with high pressure water jet: $a-3 D$ view, $b$ - front view, $c-$ side view

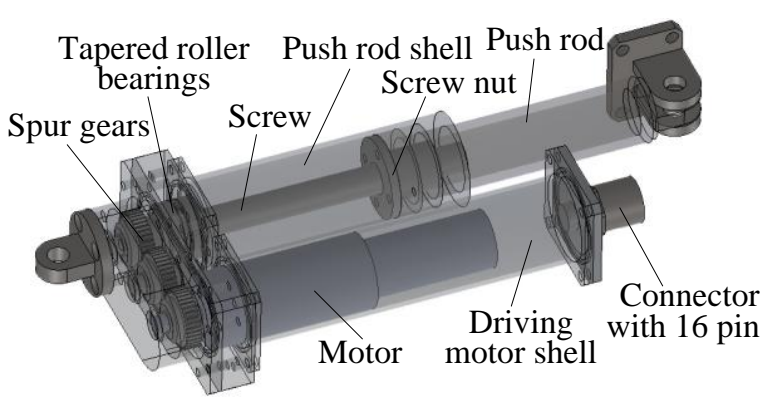

a
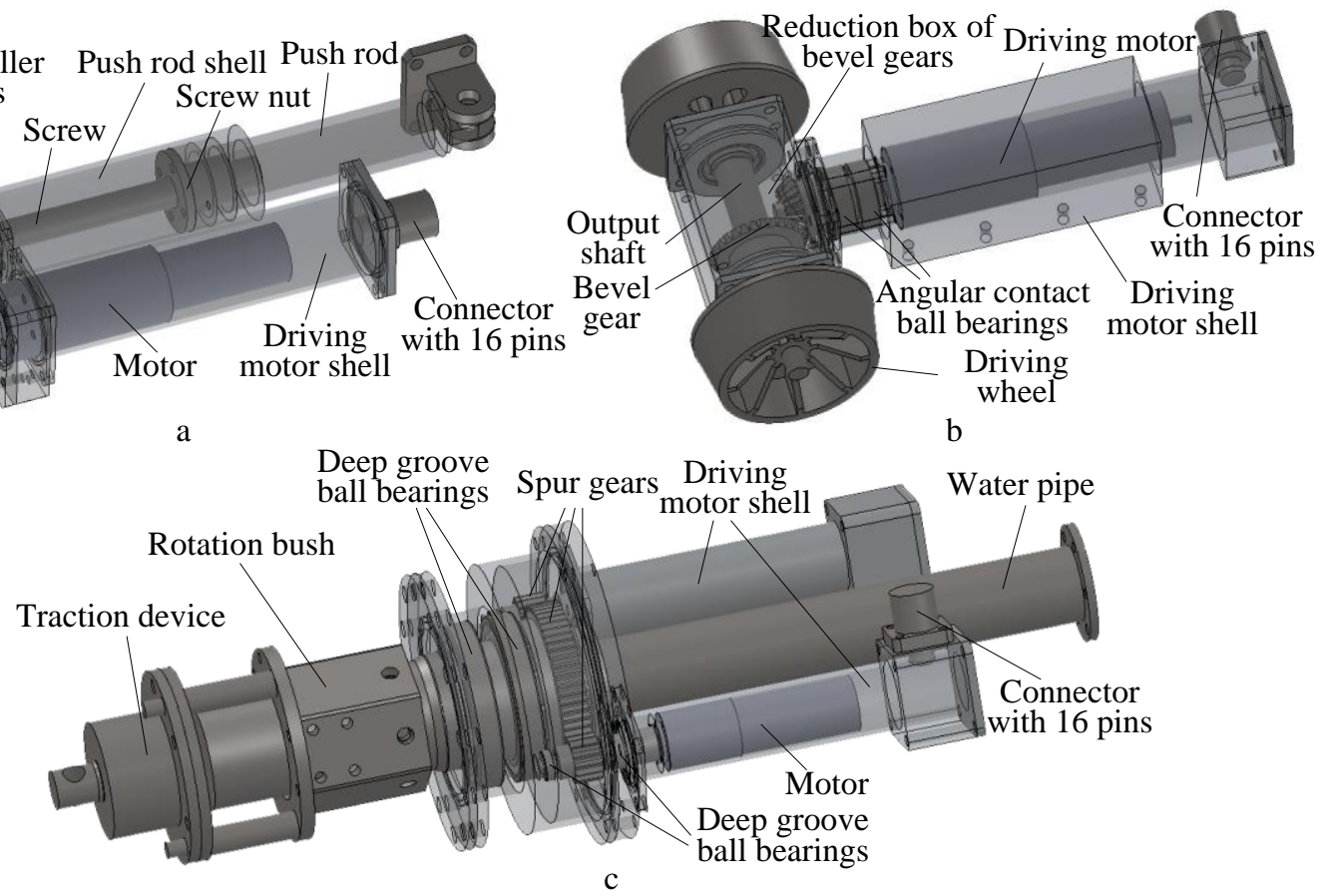

Fig. 4 Internal Structure of main parts: $a$ - screw-nut mechanism applied to drive front/rear sliding frame, $b$ - driving motor cabin and reduction gear box, $\mathrm{c}$ - mechanism applied to drive rotation arm 
Fig. 4 is the internal structure of some main parts of the IPCR. Fig. 4, $\mathrm{b}$ is the internal structure of driving motor cabin and reduction gear box, and driving torque is developed for driving wheels by reduction transmission of a pair of bevel gears. Also, Fig. 4, c is the internal structure of mechanism applied to drive rotation arm. Rotation bush fitted with rotation arm can be rotated by reduction transmission of spur gears.

\section{Traction force analysis of the IPCR}

When the IPCR is running inside the water pipelines, its output of traction force depends on the pressure exerted on the driving wheels and friction factor between the driving wheel and pipe wall; yet the self-weight of the IPCR is relatively small, preload mechanism is applied to ensure the full contact between driving wheels and pipe wall, realizing proper operation of the IPCR and exporting enough traction force $[16,17]$.

\subsection{Mechanical principle analysis of preload mechanism}

Fig. 5 is the free-body diagram of preload mechanism applied by the IPCR. With the symmetry of preload mechanism, only a set of driving wheel was analyzed below. By screw-nut mechanism driven by motor, the front/rear sliding frame will move forward along pull rods to produce the tension force $F$. The force $F$ will compress the tension spring, producing the spring force $F_{s}$ on the hinge joint $D$. Finally, three sets of driving wheels are evenly distributed around the circumference through links $B C, C D$ and $C B$ and are in full contact with the water pipe, producing the normal pressure $F_{n}$ and fraction force $F_{t}$. Supposing the IPCR moves along the water pipelines with uniform velocity, and preload mechanism can therefore be considered to be at rest and be analyzed further, where the body of IPCR is fixed [18].

As shown in Fig. 5, the coordinate Oxy is set at the centerline of the water pipelines. The $x y$ plane coincides with the symmetrical cross section of the water pipelines while $y$ axis passes through the hinge joint $C$. Where $T$ is the driving torque exerted on the driving wheels; $r_{d}$ is the radius of the driving wheels; $\alpha, \beta$ and $\gamma$ are the structure angle of preload mechanism respectively; $L, L_{1}$ and $L_{2}$ are the length of links $B C, C D$ and $D B$ respectively; $H$ is the distance from hinge joint $C$ to $x$ axis.

The constraint equations of hinge joint $A$ and $B$ are established as follows:

$$
\left\{\begin{array}{l}
x_{A}=\left(L_{0}-\Delta x\right) \cos (\alpha)+L_{1} \cos (\gamma) \\
x_{B}=L \cos (\beta) \\
y_{B}=L \sin (\beta)+H \\
\left(L_{0}-\Delta x\right) \sin (\alpha)=L_{1} \sin (\gamma) \\
\beta=\gamma+\angle B C D
\end{array},\right.
$$

where:

$$
\beta=\arcsin \frac{d-2 H-2 r_{d}}{2 L},
$$

$$
\gamma=\beta-\arccos \frac{L^{2}+L_{1}^{2}-L_{2}^{2}}{2 L L_{1}},
$$

where: $d$ is the inner diameter of the water pipelines; and $L_{0}$, $\Delta x$ are the original length and amount of compression of the tension springs respectively.

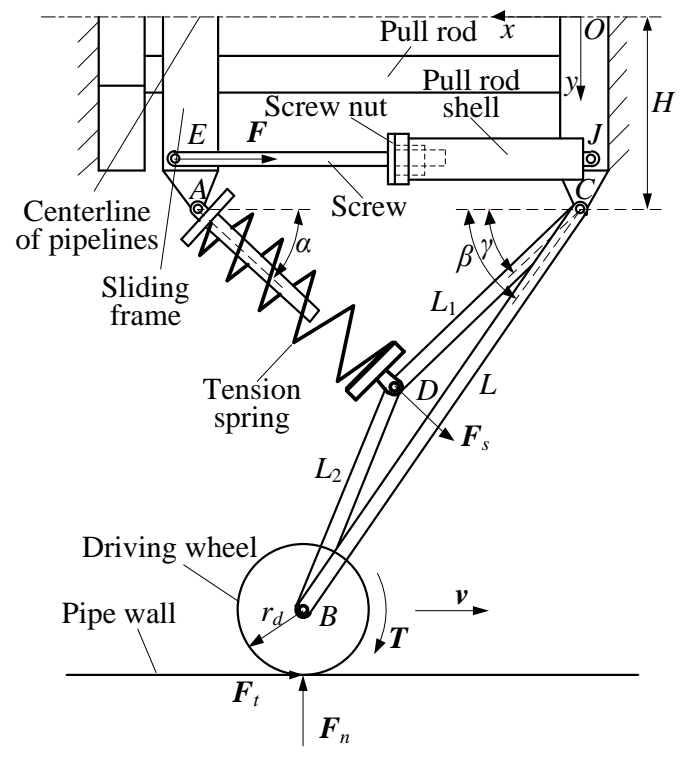

Fig. 5 Free-body diagram of preload mechanism

According to the principle of virtual work, the system of preload mechanism which is in equilibrium at this position is given an arbitrary virtual displacement, the net work done by the external forces (including the normal pressure $F_{n}$, fraction force $F_{t}$ and spring force $F_{s}$ ) during the displacement is zero [19], i.e.:

$$
\sum \delta W_{F_{i}}=F_{n} \delta y_{B}+F_{s} \delta x_{A} \cos \alpha-F_{t} \delta x_{B}=0 .
$$

Eq. (1) can be differentiated and the following equations are obtained:

$$
\left\{\begin{array}{l}
\delta x_{A}=-L_{1}(\tan (\alpha) \cos (\gamma)+\sin (\gamma)) \delta \beta \\
\delta x_{B}=-L \sin (\beta) \delta \beta \\
\delta y_{B}=L \cos (\beta) \delta \beta
\end{array} .\right.
$$

A relation exists between the fraction force $F_{t}$ and normal pressure $F_{n}$, the spring force $F_{s}$ and compression of tension spring $\Delta x$. And the relation can be expressed as:

$$
\begin{aligned}
& F_{t}=\mu F_{n}, \\
& F_{s}=k \Delta x,
\end{aligned}
$$

where: $\mu$ is the fraction coefficient between the driving wheels and water pipelines wall; $k$ is the stiffness coefficient of the tension springs.

Also, a relation exists between the spring force $F_{s}$ and the tension force $F$. And the relation can be expressed as:

$$
F_{s}=F \cos \alpha
$$


Substituting Eqs. (5)-(7) into Eq. (4), the normal pressure $F_{n}$ developed by the preload mechanism can be obtained:

$$
F_{n}=\frac{L_{1}}{L} \frac{k(\tan \alpha \cos \gamma+\sin \gamma) \cos \alpha}{\cos \beta+\mu \sin \beta} \Delta x .
$$

\subsection{Analysis of traction force}

To realize proper operation of the IPCR and export enough traction force, we must guarantee the adequate normal pressure $F_{n}$ between driving wheels and water pipelines wall. The normal pressure depends on the force developed by the preload mechanism and the self-weight of the ICPR [20]. The normal force generated by self-weight of the IPCR under the condition of the full contact between driving wheels and pipe wall will be analyzed below. Supposing the axis of the IPCR is parallel to the pipelines' axis, while the mass of the IPCR is not uniformly distributed so that the gravity shared by the front wheels and rear wheels is inconsistent. We therefore firstly studied the gravity shared by the front wheels and rear wheels respectively.

Fig. 6 is the free-body diagram of the cross section of the driving wheels when the attitude angle of the IPCR inside the pipelines is $\varphi$. As shown in Fig. 6, the coordinate $O x y$ is set at the intersection point of the cross section of the driving wheels and the centerline of the water pipelines. The $x y$ plane coincides with the cross section of the driving wheels while $x$ axis is horizontal. Where $F_{n G i}(i=1,2, \ldots, 6)$ is the normal force developed by the gravity of the IPCR and exerted on each wheel; $G_{f}$ and $G_{r}$ are the gravity shared by the front wheels and rear wheels respectively while $G_{f}^{\prime}$ and $G_{r}^{\prime}$ are the reaction force of $G_{f}$ and $G_{r}$ respectively; $s$ is the distance between the front wheel and rear wheel; $\theta$ ( $0^{\circ} \leq \theta \leq 90^{\circ}$ ) is the obliquity angle of the pipelines; $s_{1}$ and $s_{2}$ are the distance from the front/rear wheel to the gravity center of the IPCR respectively.

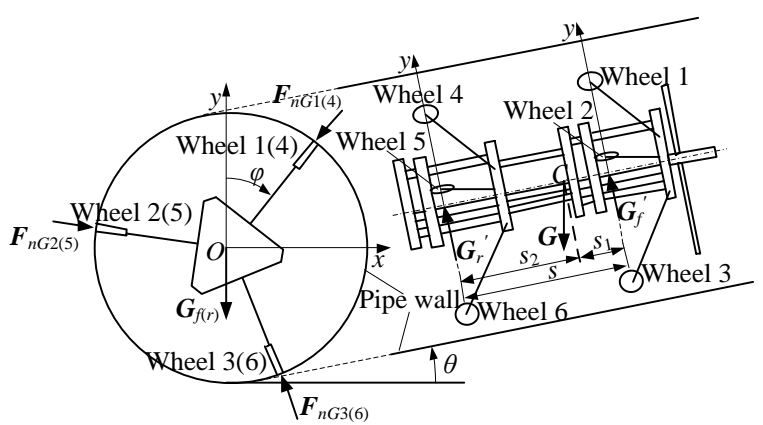

Fig. 6 Free-body diagram of the cross section of the driving wheels

According to the equilibrium condition of general planar force system, equilibrium equations within the vertical section of pipelines can be obtained:

$$
\begin{aligned}
& \sum F_{y}=0, G_{f}+G_{r}-G \cos \theta=0, \\
& \sum M_{C}=0, G_{f}^{\prime} s_{1}-G_{r}^{\prime} s_{2}=0
\end{aligned}
$$

where:

$$
\begin{aligned}
& s=s_{1}+s_{2}, \\
& G_{f}^{\prime}=G_{f}, G_{r}^{\prime}=G_{r},
\end{aligned}
$$
be obtained:

By solving Eqs. (11)-(13), the force $G_{f}$ and $G_{r}$ can

$$
\left\{\begin{array}{l}
G_{f}=\frac{s_{2}}{s} G \cos (\theta) \\
G_{r}=\frac{s_{1}}{s} G \cos (\theta)
\end{array} .\right.
$$

Also, equilibrium equations within the horizontal section of pipelines can be obtained:

$$
\begin{aligned}
& \sum F_{x}=0, F_{n G 1(4)} \sin (\varphi)+F_{n G 2(5)} \sin \left(\varphi-120^{\circ}\right)+ \\
& +F_{n G 3(6)} \sin \left(\varphi+120^{\circ}\right)=0, \\
& \sum F_{y}=0, F_{n G 1(4)} \cos (\varphi)+F_{n G 2(5)} \cos \left(\varphi-120^{\circ}\right)+ \\
& +F_{n G 3(6)} \sin \left(\varphi+120^{\circ}\right)=0,
\end{aligned}
$$

where: $\varphi$ is the attitude angle of the IPCR inside the pipelines, and the direction of increasing $\varphi$ is the clockwise direction.

Owing to the structural symmetry of the IPCR, the normal force exerted on the driving wheels only need to be analyzed with the attitude angle $\varphi$ ranged from $-30^{\circ}$ to $90^{\circ}$. The normal force generated by the gravity of the IPCR and exerted on the top driving wheel is zero within the range of certain attitude angle [21].

When $-30^{\circ} \leq \varphi \leq 60^{\circ}, F_{n G 1(4)}=0$. Substituting it into Eqs. (15) and (16), and the following equations can be obtained:

$$
\left\{\begin{array}{l}
F_{n G 2(5)}=-\frac{G_{f(r)} \sin \left(\varphi+120^{\circ}\right)}{\sin \left(240^{\circ}\right)} \\
F_{n G 3(6)}=\frac{G_{f(r)} \sin \left(\varphi-120^{\circ}\right)}{\sin \left(240^{\circ}\right)}
\end{array} .\right.
$$

Also, when $60^{\circ} \leq \varphi \leq 90^{\circ}, F_{n G 2(5)}=0$. Substituting it into Eqs. (15)-(16), and the following equations can be obtained:

$$
\left\{\begin{array}{l}
F_{n G 1(4)}=-\frac{G_{f(r)} \sin \left(\varphi+120^{\circ}\right)}{\sin \left(120^{\circ}\right)} \\
F_{n G 3(6)}=\frac{G_{f(r)} \sin \varphi}{\sin \left(120^{\circ}\right)}
\end{array} .\right.
$$

When each part of the IPCR is assembled together well and the IPCR is put to the initial position of the water pipelines, the springs on the preload mechanism are compressed in a certain extent because of the self-weight of the IPCR. Therefore, initial preloading force of the preload mechanism can just support the self-weight of the IPCR when all the swing rods are fully opened and the top driving 
wheels are just in contact with the pipelines wall. Substituting Eqs. (8) and (17)-(18) into Eq. (9), the initial preloading force generated by the preload mechanism of each spring can be obtained:

$$
\text { If }-30^{\circ} \leq \varphi \leq 60^{\circ} \text {, }
$$

$$
\left\{\begin{array}{l}
F_{s 1(4)}^{0}=0 \\
F_{s 2(5)}^{0}=-k^{\prime} \frac{G_{f(r)} \sin \left(\varphi+120^{\circ}\right)}{\sin \left(240^{\circ}\right)}, \\
F_{s 3(6)}^{0}=k^{\prime} \frac{G_{f(r)} \sin \left(\varphi-120^{\circ}\right)}{\sin \left(240^{\circ}\right)}
\end{array}\right.
$$

where: $F_{s i}^{0}(i=1,2, \ldots, 6)$ is the initial preloading force needed for each spring, and:

$$
k^{\prime}=\frac{L}{L_{1}} \frac{\cos (\beta)+\mu \sin (\beta)}{(\tan \alpha \cos \gamma+\sin \gamma) \cos (\alpha)} .
$$

$$
\text { Also, if } 60^{\circ} \leq \varphi \leq 90^{\circ} \text {, }
$$

$$
\left\{\begin{array}{l}
F_{s 1(4)}^{0}=-k^{\prime} \frac{G_{f(r)} \sin \left(\varphi+120^{\circ}\right)}{\sin \left(120^{\circ}\right)} \\
F_{s 2(5)}^{0}=0 \\
F_{s 3(6)}^{0}=k^{\prime} \frac{G_{f(r)} \sin \varphi}{\sin \left(120^{\circ}\right)}
\end{array} .\right.
$$

So from the Eqs. (14) and (19)-(21), the total initial preloading force needed for supporting the self-weight of the IPCR can be obtained:

$$
F_{s f(r) 0}=\sum F_{s i}^{0}=\left\{\begin{array}{l}
k^{\prime \prime} G \cos \varphi,-30^{\circ} \leq \varphi \leq 60^{\circ} \\
k^{\prime \prime} G \sin \left(\varphi-30^{\circ}\right), 60^{\circ} \leq \varphi<90^{\circ}
\end{array},\right.
$$

where:

$$
k^{\prime \prime}=\frac{2 L}{L_{1}} \frac{s_{2(1)}}{s} \frac{(\cos \beta+\mu \sin \beta) \cos (\theta)}{(\mathrm{t} a n \alpha \cos \gamma+\sin \gamma) \cos (\alpha)},
$$

In Eq. (22), $F_{s f(r) 0}$ is the total initial preloading force of the three front/rear springs and is used for supporting the self-weight of the IPCR.

Considering the type of friction between the driving wheels and pipe wall is rolling friction, so their friction resistance can be neglected. Therefore, from Eqs. (7)-(8), (10) and (22)-(23), the total traction force $F_{T}$ of the IPCR can be obtained:

$$
F_{T}=\frac{\mu}{k^{\prime}}\left[\left(F_{s f}-F_{s f 0}\right)+\left(F_{s r}-F_{s r 0}\right)\right]
$$

where: $F_{s f}$ and $F_{s r}$ are the total spring preloading force of all the front and rear springs respectively.

From Eqs. (19)-(21) can be seen, when all the swing rods are fully opened and the top driving wheels are just in contact with the pipelines wall, the initial preloading force values of each spring needed for supporting the swing rods is different. However, due to the structural symmetry of the IPCR and the constraint between the front/rear sliding frame and pull rods, the front/rear sliding frame driven by screw-nut mechanism moves forward to develop three tension forces $F$, whose values is identical. Considering the factors above, we knew each spring force is different, which will lead to the axis of the IPCR is not parallel to the pipelines' axis, exacerbating the self-deformation of the IPCR and increasing the power consumption. To solve this problem and increase simultaneously the total traction force of the IPCR, the mode of multi-motor drive was used in our design and six motors finally realize the power balance through control algorithm.

Besides, from Eqs. (19)-(21) we can know, if the attitude angle $\varphi=0^{\circ}$, the following equations can be obtained:

$$
F_{s 1(4)}^{0}=0, F_{s 2(5)}^{0}=F_{s 3(6)}^{0}=k^{\prime} G_{f(r)},
$$

Also, if the attitude angle $\varphi=60^{\circ}$, the following equations can be obtained:

$$
F_{s 1(4)}^{0}=F_{s 2(5)}^{0}=0, F_{s 3(6)}^{0}=k^{\prime} G_{f(r)},
$$

From Eqs. (25)-(26), when the IPCR enters the initial position of the pipelines, the typical attitude $\left(\varphi=0^{\circ}\right.$ or $\left.\varphi=60^{\circ}\right)$ of the IPCR can be adopted to reduce the self-deformation.

\subsection{Necessity analysis of initial preloading force}

As we can know from the results described above, when each part of the IPCR is assembled together well and the IPCR is put to the initial position of the water pipelines, all the springs of the preload mechanism must be exerted preloading force, which is used for not only supporting the self-weight of the IPCR but also providing the normal force for the generation of traction force. Supposing when the IPCR just enters the initial position of the pipelines, all the springs of the preload mechanism are not compressed and are in the state of original length. In this extreme case, it will lead to vibration of the IPCR caused by the misdistribution mass and the mass center does not coincide with the geometrical center.

According to the actual condition and performance requirements, any vibration of an object can be simplified as different vibration model [22]. Therefore, without considering the self-elastic deformation of the IPCR and unspring mass and taking the IPCR as a bar (rigid body), the vibration of the IPCR in vertical plane can be simplified as damped vibration of the system with two degrees of freedom [22], as shown in Fig. 7. Where $C$ is the mass center of the bar, which does not coincide with the geometrical center; $l$ is the bar lengths; $d$ is the distance between two springs; $l_{1}$, $l_{2}\left(l_{1} \neq l_{2}\right)$ is the distance between spring and mass center respectively, and $l_{3}, l_{4}\left(l_{3} \neq l_{4}\right)$ is the distance between bar end and adjacent spring respectively; $k_{1}, k_{2}$ is the equivalent stiffness coefficient of front and rear springs, and $c_{1}, c_{2}$ is the equivalent damping coefficient of damper. Taking equilib- 
rium position of the system as the origin, and only the displacement of the vertical direction of the vibration system and the pitch vibration around the mass center was considered. So the displacement $x_{C}$ of mass center and angle displacement $\psi$ around the mass center (the direction of increasing $\psi$ is the clockwise direction) was defined respectively as the generalized coordinates of the vibration system. The restitution force $F_{1}$ and $F_{2}$ exerted on the system and the displacement $x_{1}$ and $x_{2}$ are opposite in direction, so the force $F_{1}$ and $F_{2}$ can be expressed as:

$$
F_{1}=k_{1} x_{1}, F_{2}=k_{2} x_{2} \text {. }
$$

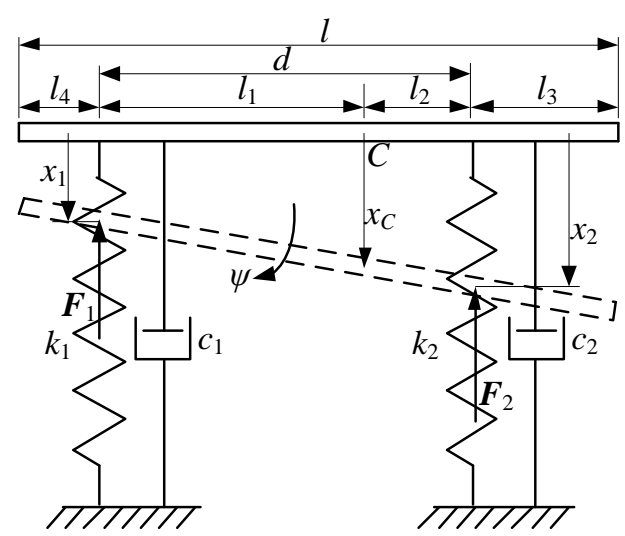

Fig. 7 Sketch of simplified vibration model

At this point, the coordinate of mass center $x_{C}$ can be expressed as:

$$
x_{C}=x_{1}+\frac{l_{1}}{d}\left(x_{2}-x_{1}\right)
$$

And the slight angle displacement $\psi$ around the mass center can be expressed as:

$$
\psi=\frac{x_{2}-x_{1}}{d}
$$

According to the differential equation of plane motion of a rigid body [19], the differential equation of plane motion of the system can be obtained:

$$
\left\{\begin{array}{l}
m \ddot{x}_{C}=-F_{1}-F_{2}-c_{1} \dot{x}_{1}-c_{2} \dot{x}_{2} \\
J_{C} \ddot{\psi}=\left(F_{1}+c_{1} \dot{x}_{1}\right) l_{1}-\left(F_{2}+c_{2} \dot{x}_{2}\right) l_{2}
\end{array},\right.
$$

where: $m$ is the mass of the bar; $J_{C}$ is the moment of inertia of the bar around the axis which is through the mass center and is perpendicular to the motion plane. are identical, so:

Considering all the springs mounted on the IPCR

$$
\begin{aligned}
& k_{1}=k_{2}, c_{1}=c_{2}, \\
& d=l_{1}+l_{2} .
\end{aligned}
$$

Substituting Eqs. (27)-(29) and (31)-(32) into the Eq. (30), the vibration equations of the simplified vibration system of the IPCR can be obtained:

$$
\left\{\begin{array}{l}
m \ddot{x}_{C}+2 c_{1} \dot{x}_{C}-c_{1}\left(l_{1}-l_{2}\right) \dot{\psi}+ \\
+2 k_{1} x_{C}-k_{1}\left(l_{1}-l_{2}\right) \psi=0 \\
J_{C} \ddot{\psi}+c_{1}\left(l_{2}-l_{1}\right) \dot{x}_{C}+c_{1}\left(l_{1}^{2}+l_{2}^{2}\right) \dot{\psi}+ \\
+k_{1}\left(l_{2}-l_{1}\right) x_{C}+k_{1}\left(l_{1}^{2}+l_{2}^{2}\right) \psi=0
\end{array} .\right.
$$

low:

Eqs. (33) can be simplified to matrix form as fol-

$$
M \ddot{U}+C \dot{U}+K U=0
$$

When time $t=0$, the initial conditions of the simplified vibration system are as follow:

$$
x_{C 0}=-\frac{m g}{2 k_{1}}, \dot{x}_{C 0}=0 ; \psi_{0}=0, \dot{\psi}_{0}=g \text {, }
$$

where: $g$ is the acceleration of gravity. And in Eq. (34),

$$
\begin{aligned}
& U=\left[\begin{array}{l}
x_{C} \\
\psi
\end{array}\right], \\
& M=\left[\begin{array}{ll}
m & \\
& J_{C}
\end{array}\right], \\
& C=\left[\begin{array}{cc}
2 c_{1} & c_{1}\left(l_{2}-l_{1}\right) \\
c_{1}\left(l_{2}-l_{1}\right) & c_{1}\left(l_{1}^{2}+l_{2}^{2}\right)
\end{array}\right], \\
& K=\left[\begin{array}{cc}
2 k_{1} & k_{1}\left(l_{2}-l_{1}\right) \\
k_{1}\left(l_{2}-l_{1}\right) & k_{1}\left(l_{1}^{2}+l_{2}^{2}\right)
\end{array}\right],
\end{aligned}
$$

where: $U$ is the generalized coordinate matrix of the system, $M$ is the mass matrix of the system, $C$ is the damping matrix of the system, $K$ is the stiffness matrix of the system.

From the analysis above, we conclude the vibration of the IPCR in vertical plane can be simplified as damped vibration of the system with two degrees of freedom and its vibration equations is as shown in Eq. (34). According to the mechanical vibration theory, the vibration of the IPCR belongs to free vibration with viscous damping, and its initial excitation is the self-weight of the IPCR.

As we know, the system with slight damping is damped free vibration while the system with large damping is not damped free vibration but exponential attenuation motion, which will attenuate to zero in a short time [23]. However, no matter what vibration form the IPCR is, the IPCR may fail to start properly to move forward and the high pressure water jet cleaning device cannot work properly in initial position of the pipelines, which is most likely to cause the cleaning system crash. Therefore, its initial excitation caused the self-weight of the IPCR will be weaken by adding initial preloading force of all springs, and this is the most efficient way to ensure proper operation of the IPCR.

Also, from the analysis of traction force, when the attitude angle of the IPCR is $0^{\circ}$, the deformation of all springs is relatively small; besides, the equivalent stiffness coefficient $k_{1}$ and $k_{2}$ of simplified vibration system becomes relatively larger, which can reduce vibration of the IPCR. 
So setting the attitude angle $\varphi=0^{\circ}$ is in initial position also an efficient way to ensure proper operation of the IPCR.

\section{Obstacle-overcoming capability analysis of the IPCR}

To ensure the traveling capability of the IPCR, in our design, the mode of multi-motor drive was used for increasing the total traction force of the IPCR and six motors can be controlled separately. And front/rear sliding frame which can move forward/backward along three pull rods was applied for improving the pipelines adaptability and obstacle-overcoming capability of the IPCR. All possible obstacles inside pipelines may stop the IPCR from proper operation, so obstacle-overcoming is a necessary capability of the IPCR.

\subsection{Mechanical model of obstacle-overcoming}

In this paper, only the typical obstacle inside pipelines is analyzed due to length limitations, i.e. the change of diameters. Also, supposing the IPCR moves along the water pipelines with uniform velocity and the axis of the IPCR keeps in the horizontal direction before meeting the obstacle. Fig. 8 is the mechanical model of obstacle-overcoming. Where $F_{n \lambda i}(i=1,2,3$ or $4,5,6)$ is the normal pressure and $F_{t \lambda i}(i=1,2,3$ or $4,5,6)$ is the fraction force exerted on driving wheel at contact point; $\lambda$ is the angle of obstacle-overcoming and $\theta\left(0^{\circ} \leq \theta \leq 90^{\circ}\right)$ is the obliquity angle of the pipelines; $h$ is the height of obstacle-overcoming; $r_{d}$ is the radius of driving wheel.

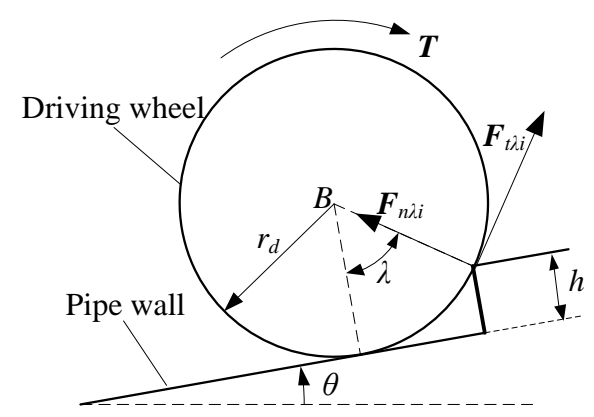

Fig. 8 Mechanical model of obstacle-overcoming (diameter becomes smaller)

The normal pressure and fraction force exerted on three front/rear driving wheels will be changed at the moment of contacting the obstacle. From Eq. (9), the normal pressure $F_{n \lambda i}$ of obstacle-overcoming wheel without sliding can be obtained:

$$
F_{n \lambda i}=\frac{L_{1}}{L} \frac{k(\tan (\alpha) \cos (\gamma)+\sin (\gamma)) \cos (\alpha)}{\cos (\lambda+\beta)+\mu \sin (\lambda+\beta)} \Delta x
$$

In Eq. (40):

$$
\lambda=\arccos \left(1-\frac{h}{r_{d}}\right)
$$

If the wheels of obstacle-overcoming are the front (rear) three driving wheels, and we know the traction force of rear (front) three driving wheels keeps constant. Supposing the force $G_{f}\left(G_{r}\right)$ shared by front (rear) driving wheels is ignored at the moment of contacting the obstacle, while the total traction force of rear (front) driving wheels is still calculated according to Eq. (24). So when the IPCR can succeed in overcoming obstacle, the mechanical equation must be met:

$$
\sum\left(F_{t \lambda i} \cos (\lambda)-F_{n \lambda i} \sin (\lambda)\right)+F_{T}^{\prime}-F_{L} \geq 0
$$

where: $F_{T}^{\prime}$ can be obtained according to Eq. (24); $F_{L}$ is the total load on the IPCR.

$$
\begin{aligned}
& F_{T}^{\prime}=\left\{\begin{array}{l}
\frac{\mu}{k^{\prime}}\left[\left(F_{s r}-F_{s r 0}\right)\right], i=1,2,3 \\
\frac{\mu}{k^{\prime}}\left[\left(F_{s f}-F_{s f 0}\right)\right], i=4,5,6
\end{array},\right. \\
& F_{L}=\mu_{1} u L_{W} g \cos \theta+F_{I}+G \sin \theta .
\end{aligned}
$$

In Eq. (44), $\mu_{1}\left(\mu_{1}=0.06\right)$ is the resistance coefficient between rolling bracket and pipe wall; $u(u=3 \mathrm{~kg} / \mathrm{m})$ is the total mass per unit length of cables and water pipe; $L_{W}$ $\left(0 \leq L_{W} \leq 1600 \mathrm{~m}\right)$ is the length of cables and water pipe pulled by the IPCR; $g$ is the gravity acceleration; $F_{I}$ is the rolling friction resistance of the IPCR.

After the IPCR succeeds in overcoming obstacle, the total traction force of front (rear) driving wheels can be obtained by replacing $d$ with $(d-2 h)$ in Eq. (24). Obviously the total traction force becomes large, so front (rear) sliding frame should move backward properly to reduce the deformation of front (rear) springs. Also, after the IPCR succeeds in overcoming obstacle (diameter becomes larger), front (rear) sliding frame should move forward properly to increase the deformation of front (rear) spring, providing enough traction force for the IPCR.

\subsection{Improvement of obstacle-overcoming}

In Eq. (34), it was established based on the two generalized coordinates $x_{C}$ and $\psi$, and they are principal coordinates of the vibration system. Also, the displacement $x_{1}$ and $x_{2}$ can be defined as the generalized coordinates of the vibration system [23]. Substituting Eqs. (27)-(29) and (31)(32) into the Eqs. (30), and Eqs. (33) can also be expressed as:

$$
\left\{\begin{array}{l}
\frac{m l_{2}}{d} \ddot{x}_{1}+\frac{m l_{1}}{d} \ddot{x}_{2}+c_{1} \dot{x}_{1}+c_{1} \dot{x}_{2}+k_{1} x_{1}+k_{1} x_{2}=0 \\
-\frac{J_{C}}{d} \ddot{x}_{1}+\frac{J_{C}}{d} \ddot{x}_{2}-c_{1} l_{1} \dot{x}_{1}+c_{1} l_{2} \dot{x}_{2}-k_{1} l_{1} x_{1}+k_{1} l_{2} x_{2}=0
\end{array},\right.
$$

when time $t=0$, the initial conditions of the vibration system are as follow:

$$
x_{10}=x_{20}=-\frac{m g}{2 k_{1}}, \dot{x}_{10}=\dot{x}_{20}=0 \text {. }
$$

In Eqs. (45), $x_{1}$ and $x_{2}$ can be eliminated after proper transformation, and the following equations can be obtained: 


$$
\left\{\begin{array}{l}
m \frac{l_{2}^{2}+\rho_{C}^{2}}{d^{2}} \ddot{x}_{1}+m \frac{l_{1} l_{2}-\rho_{C}^{2}}{d^{2}} \ddot{x}_{2}+c_{1} \dot{x}_{1}+k_{1} x_{1}=0 \\
m \frac{l_{1}^{2}+\rho_{C}^{2}}{d^{2}} \ddot{x}_{2}+m \frac{l_{1} l_{2}-\rho_{C}^{2}}{d^{2}} \ddot{x}_{1}+c_{1} \dot{x}_{2}+k_{1} x_{2}=0
\end{array},\right.
$$

where: $\rho_{C}$ is the gyration radius of the bar around the axis of mass center.

$$
\rho_{C}=\frac{J_{C}}{m}
$$

Next, we will analyze the vibration transmission between front and rear springs. For the convenience of study, the damping of the vibration system is ignored, so Eqs. (47) can be simplified as follow:

$$
\left\{\begin{array}{l}
\ddot{x}_{1}+\eta_{1} \ddot{x}_{2}+\omega_{1}^{2} x_{1}=0 \\
\ddot{x}_{2}+\eta_{2} \ddot{x}_{1}+\omega_{2}^{2} x_{2}=0
\end{array},\right.
$$

where: $\eta_{1}, \eta_{2}$ is the coefficient of association which indicates the relationship of $x_{1}$ and $x_{2} ; \omega_{1}, \omega_{2}$ is the offset frequency which indicates the vibration frequency of independent vibration of front/rear springs. And if $x_{1}=0$, the vibration frequency of the system is $\omega_{2}$, while if $x_{2}=0$, the vibration frequency of the system is $\omega_{1}$.

$$
\begin{aligned}
& \eta_{1}=\frac{l_{1} l_{2}-\rho_{C}^{2}}{l_{2}^{2}+\rho_{C}^{2}}, \eta_{2}=\frac{l_{1} l_{2}-\rho_{C}^{2}}{l_{1}^{2}+\rho_{C}^{2}} \\
& \omega_{1}=\sqrt{\frac{k_{1} d^{2}}{m\left(l_{2}^{2}+\rho_{C}^{2}\right)}}, \omega_{2}=\sqrt{\frac{k_{1} d^{2}}{m\left(l_{1}^{2}+\rho_{C}^{2}\right)}},
\end{aligned}
$$

when the IPCR working inside the pipelines, we hope the vibration of front (rear) springs of the IPCR cannot reach to rear (front) springs. So the following relation between the mass distribution of the IPCR and the position of front/rear swing rods must be met:

$$
\varepsilon=\frac{\rho_{C}^{2}}{l_{1} l_{2}}=1 .
$$

In Eq. (52), $\varepsilon$ is the coefficient of mass distribution. When $\varepsilon=1$, the coefficient of association is zero, i.e. $\eta_{1}=\eta_{2}=0$. And Eqs. (49) can be simplified as follow:

$$
\left\{\begin{array}{l}
\ddot{x}_{1}+\omega_{1}^{2} x_{1}=0 \\
\ddot{x}_{2}+\omega_{2}^{2} x_{2}=0
\end{array} .\right.
$$

Eqs. (53) shows that when $\varepsilon=1$, the natural frequency of two principal vibrations is equal to the offset frequency of front and rear springs of the IPCR, i.e.:

$$
\omega_{1}=\sqrt{\frac{k_{1}}{m^{l_{2} / d}}}, \omega_{2}=\sqrt{\frac{k_{1}}{m^{l_{1} / d}}},
$$

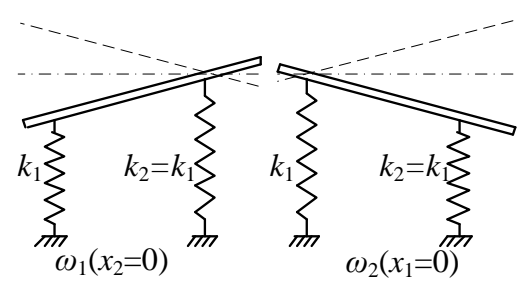

Fig. 9 The two principal vibrations (the natural frequency is equal to the offset frequency $\omega_{1}$ and $\omega_{2}$ )

Fig. 9 is two the principal vibrations with the frequency $\omega_{1}$ and $\omega_{2}$, i.e. when rear springs vibrate up and down at the frequency $\omega_{1}$, front springs keep still; when front springs vibrate up and down at the frequency $\omega_{2}$, rear springs keep still.

From Eqs. (46) and (53)-(54), the solution of Eqs. (53) can be obtained:

$$
\left\{\begin{array}{l}
x_{1}=\frac{m g}{2 k_{1}} \sin \left(\omega_{1} t-\frac{\pi}{2}\right) \\
x_{2}=\frac{m g}{2 k_{1}} \sin \left(\omega_{2} t-\frac{\pi}{2}\right)
\end{array} .\right.
$$

In the process of obstacle-overcoming, front/rear springs of the IPCR will vibrate arising from the excitation caused by obstacles. The stable output of traction force will be affected. And from Eq. (55) we know, when $\varepsilon=1$, there is no relation between the vibration of front springs and rear springs of the IPCR without one impacting the other. Therefore, the reasonable arrangement of load and parts on the IPCR should be carried out to make the coefficient of mass distribution as equal to 1 as possible. In this way, the vibration transmission between front and rear springs can be effectively avoided, which is beneficial to the improvement of traveling capability of the IPCR and the achievement of power balance control for multi-motor.

\section{Simulation}

To testify the traveling capability of the proposed IPCR, some basic simulations have been conducted.

\subsection{Simulation environment}

A virtual simulation platform including the simplified physical prototype of the IPCR and the water pipelines is established. On the platform, the water pipelines is fixed on the ground and the simplified physical prototype of the IPCR complies with the actual assembly relationship shown in Fig. 3 by the joint constraints; besides, contact constraints are used between the driving wheels of the IPCR and the pipe wall.

In the simulations, to fully verify the validity of the proposed IPCR, two groups of tests including traction force and traveling capability are implemented inside the pipelines with different parameters.

\subsection{Simulation of traction force}

In the simulation of traction force, the typical pipeline in application of engineering construction is chosen and its diameter is $1400 \mathrm{~mm}$. The pipeline is placed horizontally and is fixed on the ground. To test the maximum traction 
force of the IPCR, a virtual spring is fixed on the back end of the IPCR and the other end of the spring is fixed on the ground; besides, the maximum torque exerted on the driving wheels is the single input, and the maximum pull force is exerted on the front/rear sliding frame to generate the maximum normal force between the IPCR and the pipe wall. Fig. 10 is the obtained curves of traction force and velocity of the IPCR, where $D$ is the diameter of the pipelines.

As shown in Fig. 10, with the IPCR moving forward, the spring fixed on the back end of the IPCR is gradually stretched. As the deformation of the spring becomes larger, the velocity of the IPCR becomes faster firstly and then smaller. When the velocity direction of the IPCR changes, the maximum traction force is obtained and its value is $6865.19 \mathrm{~N}$.

Setting the rolling friction resistance $F_{I}$ of the IPCR is $1000 \mathrm{~N}$ (the coefficient of rolling friction is about 0.02), and From Eq. (44) we know, if the IPCR climbs the sloping pipelines all the time, the maximum obliquity angle $\theta_{\max }$ of pipelines the IPCR can conquer can be obtained:

$$
\theta_{\max }=43.8^{\circ}
$$

Considering the water pipelines in application of engineering construction is almost placed horizontally and from Eq. (56) we know, the proposed IPCR is powerful with traction force to guarantee its operation distance reach $1500 \mathrm{~m}$.

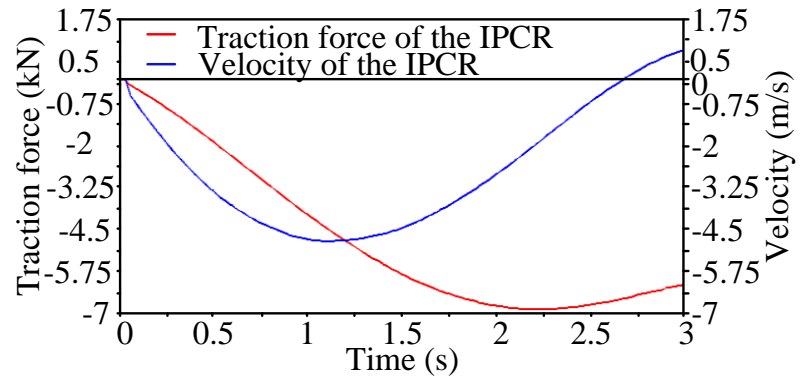

Fig. 10 Curves of traction force and velocity of the IPCR $\left(\varphi=60^{\circ}, \theta=0^{\circ}, D=1400 \mathrm{~mm}\right)$

\subsection{Simulation of traveling capability}

\subsubsection{Vibration of the IPCR in initial position}

From the necessity analysis of the initial preloading load, the IPCR without exerted initial preloading load will vibrate in initial position of the water pipelines. The way to avoid the vibration in initial position is to add initial preloading force of all springs or set the attitude angle. In the simulations, the pipeline is still placed horizontally and is fixed on the ground; besides, the IPCR is not given the torque input. Fig. 11 is the obtained curves of vibration in initial position at the typical attitude angle.
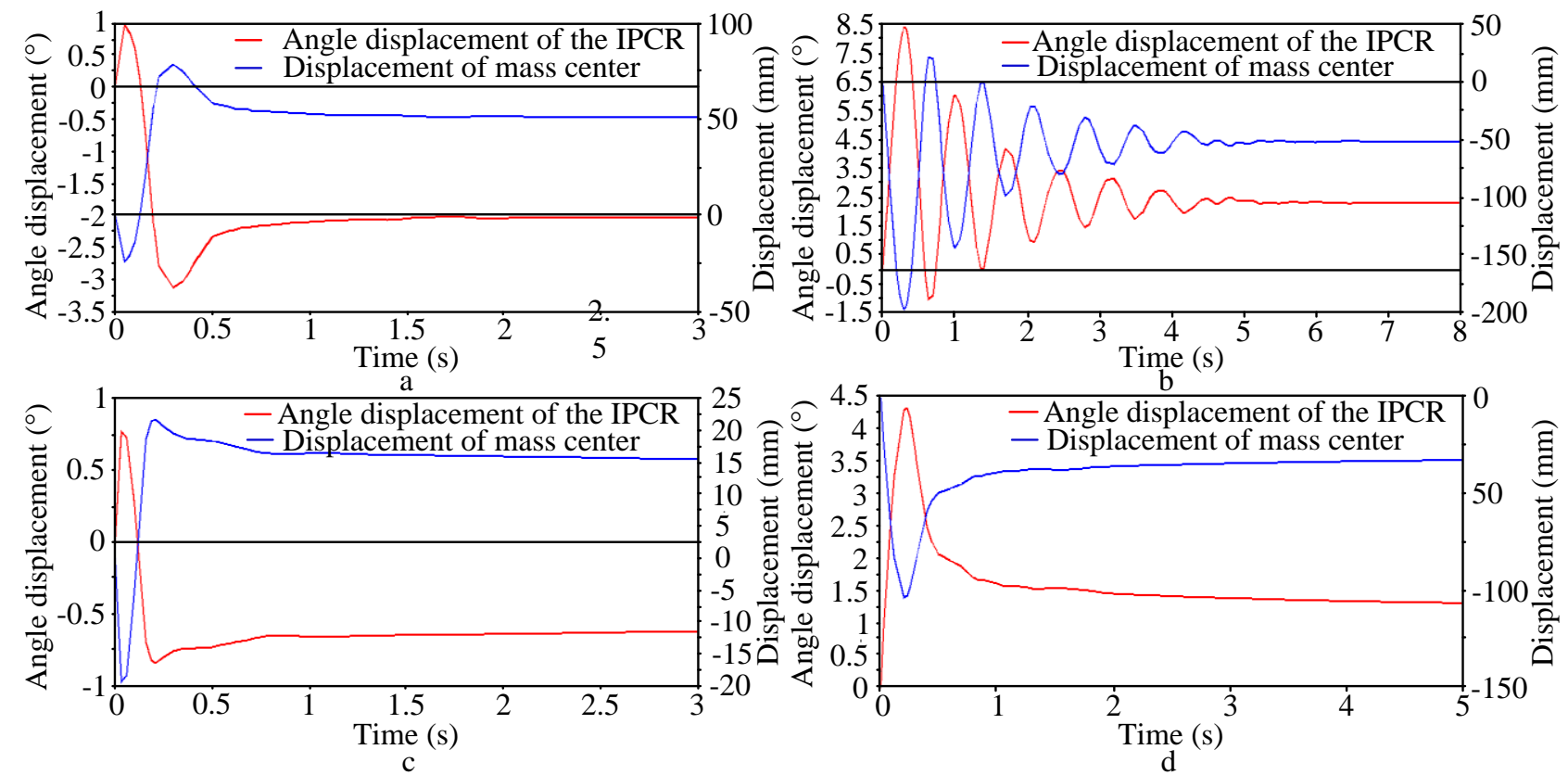

Fig. 11 Vibration of the IPCR in initial position: $\mathrm{a}-$ no added initial preloading force $\left(\varphi=0^{\circ}, \theta=0^{\circ}\right)$; $\mathrm{b}-$ no added initial preloading force $\left(\varphi=60^{\circ}, \theta=0^{\circ}\right) ; \mathrm{c}$ - added initial preloading force $\left(\varphi=0^{\circ}, \theta=0^{\circ}\right) ; \mathrm{d}-$ added initial preloading force $\left(\varphi=60^{\circ}, \theta=0^{\circ}\right)$

The results show there is almost no vibration after adding the preloading force or setting the attitude angle and the IPCR can stand still in a very short time. As shown in Fig. 11, a-b, under the condition of no added preloading force, the vibration with is more sharply and the amplitude of displacement and angle displacement is much larger than that with $\varphi=60^{\circ}$. This is because the equivalent stiffness coefficient of simplified vibration system is relatively small at $\varphi=60^{\circ}$. The reduction percentage of dis-placement and angle displacement of the IPCR is shown in Table 2.

As shown in Fig. 11, c-d, after adding initial preloading force, the vibration is not damped free vibration but exponential attenuation motion, which attenuates to zero in a short time. As the initial excitation is very small, the displacement and angle displacement of the IPCR, to a great extent, are much smaller than before (with no added initial preloading force), as shown in Table 3 . In conclusion, the above obtained results show that setting the attitude angle in 
initial position or adding initial preloading force of all springs can obviously weaken vibration of the proposed IPCR.

Table 2

Reduction percentage at two different attitude angle (no added preloading force)

\begin{tabular}{|c|c|c|c|c|}
\hline & $\begin{array}{c}\text { Maximum } \\
\text { displace- } \\
\text { ment }\end{array}$ & $\begin{array}{c}\text { Maximum } \\
\text { angle dis- } \\
\text { placement }\end{array}$ & $\begin{array}{c}\text { Final } \\
\text { dis- } \\
\text { place- } \\
\text { ment }\end{array}$ & $\begin{array}{c}\text { Final an- } \\
\text { gle dis- } \\
\text { placement }\end{array}$ \\
\hline $\begin{array}{c}\varphi=0^{\circ} \\
\text { vs. } \\
\varphi=60^{\circ}\end{array}$ & $60.5 \%$ & $62.7 \%$ & $0.64 \%$ & $12.8 \%$ \\
\hline
\end{tabular}

Table 3

Reduction percentage after adding preloading force

\begin{tabular}{|c|c|c|c|c|}
\hline & $\begin{array}{c}\text { Maximum } \\
\text { displace- } \\
\text { ment }\end{array}$ & $\begin{array}{c}\text { Maximum } \\
\text { angle dis- } \\
\text { placement }\end{array}$ & $\begin{array}{c}\text { Final } \\
\text { dis- } \\
\text { place- } \\
\text { ment }\end{array}$ & $\begin{array}{c}\text { Final an- } \\
\text { gle dis- } \\
\text { placement }\end{array}$ \\
\hline$\varphi=0^{\circ}$ & $69.5 \%$ & $69.7 \%$ & $72.2 \%$ & $73.4 \%$ \\
\hline$\varphi=60^{\circ}$ & $47.5 \%$ & $48.5 \%$ & $33.5 \%$ & $40.4 \%$ \\
\hline
\end{tabular}

\subsubsection{Obstacle-overcoming capability to suit multi-radius} pipeline of the IPCR

In the application of engineering construction, the pipelines are often positioned in different posture to meet different connection demands. So the IPCR may encounter obstacles at the pipelines connections [2]. In the simulation, we choose the straight pipeline with multi-radius. The diameter of initial part of the pipeline is not variable and is long enough to allow the IPCR to reach maximum velocity before meeting obstacles, as shown in Fig. 12. The torque exerted on the driving wheels is the single input, and the proper

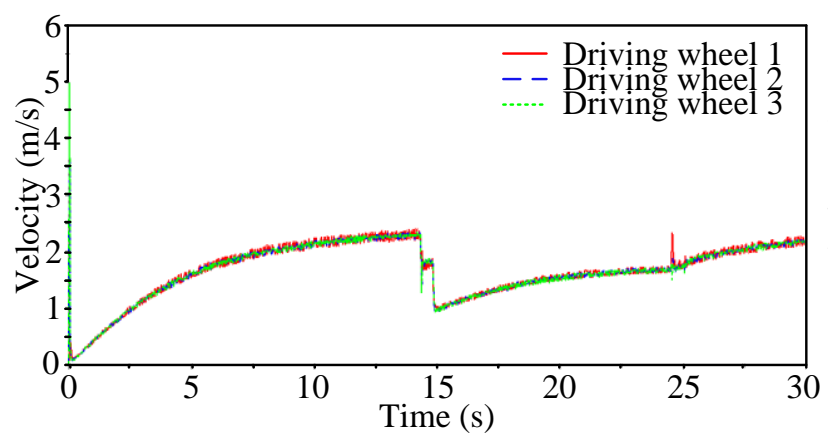

a pull force is exerted on the front/rear sliding frame to generate the preloading force.

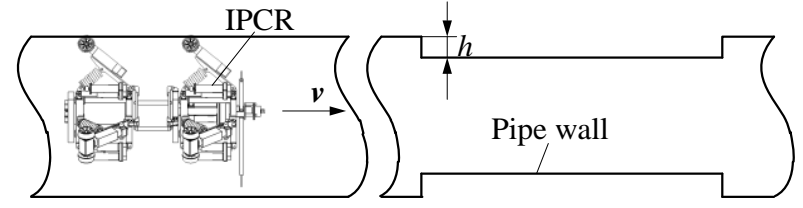

Fig. 12 Straight pipeline with multi-radius $(h=30 \mathrm{~mm}$, $\left.\varphi=0^{\circ}, \theta=0^{\circ}\right)$

Fig. 13 shows the velocity of the driving wheels, and the results indicate that the proposed IPCR can succeed in passing through the pipeline with multi-radius. As shown in Fig. 13, a-b, the velocity of all front driving wheels instantly reduces and only the velocity vibration of the bottom driving wheels occurs at the moment of contacting the obstacle; the velocity instantly reduces again at the moment of completely overcoming the obstacle. The vibration is not transferred to the rear driving wheels. Also, when the proposed IPCR jumps down the obstacle, only the velocity of the top front driving wheel vibrates and the transition of velocity is smooth. The velocity of all rear driving wheels vibrates with larger amplitude. This is because the mass center of the IPCR is forward so that the front driving wheels support the majority of the IPCR's weight; besides, the pull force exerted on the front and rear sliding frame is identical. So the normal pressure of rear driving wheels is relatively small, leading to the vibration with larger amplitude.

Fig. 14 is the force on the springs at the process of operation of the IPCR. Regardless of the front or rear springs, the beats of force on the top spring is relatively large and only the shock on the top spring is very large the moment the IPCR contacts the obstacle.

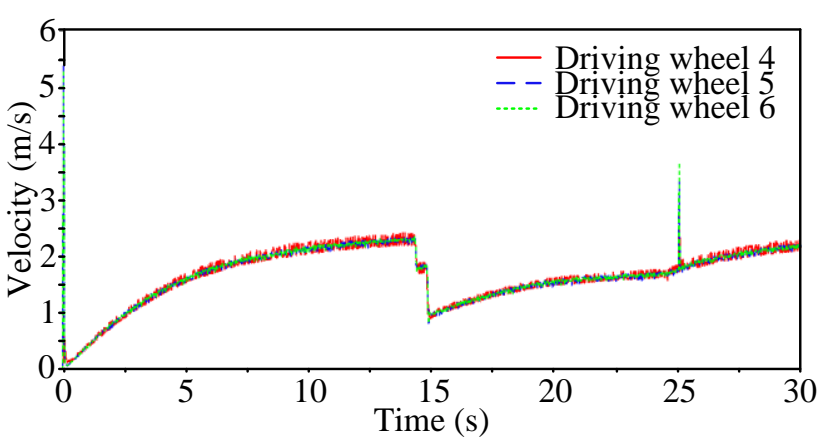

b

Fig. 13 Velocity of the driving wheels: $a$ - velocity of the front driving wheels; $b$ - velocity of the rear driving wheels

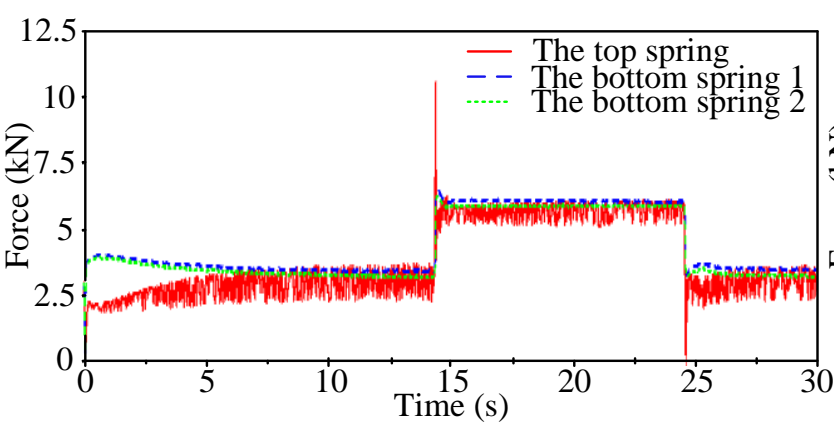

a

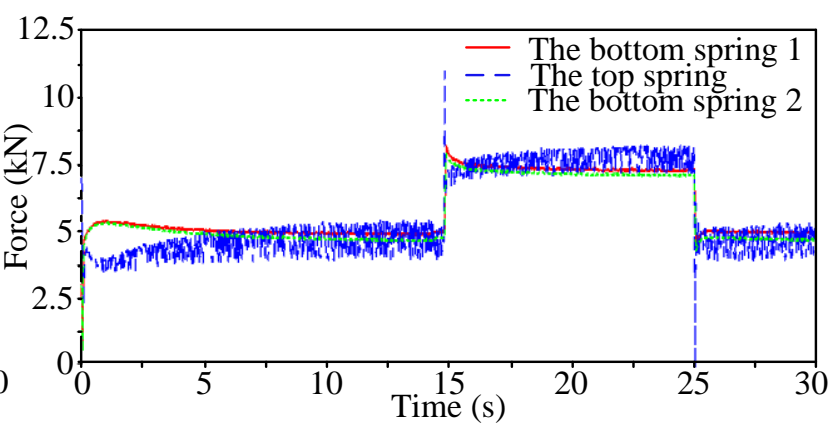

$\mathrm{b}$

Fig. 14 Force on the driving wheels: $a$ - force on the front springs; $b$ - force on the rear springs 
To sum up, the proposed IPCR has a certain capability to overcome obstacles. Vibration between the front and rear driving wheels in not transferred, because the velocity of the IPCR is small. Considering the operation speed of the IPCR is relatively slow or the obliquity angle of the pipeline is not equal to zero in real application, the IPCR needs more out-put torque to successfully overcome obstacles inside pipe-lines; and the shock on the top spring can also be decreased the moment the IPCR contacts the obstacle.

\section{Conclusions}

This paper proposed a novel in-pipe cleaning robot equipped with high pressure water jet and cleaning system of water pipelines. The whole mechanism of the IPCR was designed and the mechanisms principle of the IPCR was analyzed. The total traction force of the IPCR was given out after analyzing the mechanical principle of the preload mechanism. The capability of obstacle-overcoming was analyzed. The vibration equations of the simplified vibration system of the IPCR were obtained. On this basis, the necessity of adding initial preloading force on all springs was verified and the methods to improve the capability of obstacleovercoming were proposed. Simulations have been implemented to verify the traveling capability of the pro-posed IPCR. The results show that the IPCR is powerful with traction force and has a certain capability to overcome obstacles. The results can give a reference to the achievement of power balance control for multi-motor and real engineering application in the future.

\section{References}

1. Zhu, X.; Wang, W.; Zhang, S.; Liu, S. 2017. Experimental research on the frictional resistance of fluiddriven pipeline robot with small size in gas pipeline, Tribology Letters 65(2): 1-10. https://doi.org/10.1007/s11249-017-0830-z.

2. Li, T.; Ma, S.; Li, B.; Wang, M.; Wang, Y. 2016. Axiomatic design method to design a screw drive in-pipe robot passing through varied curved pipes, Science China Technological Sciences 59(2): 191-202. https://doi.org/10.1007/s11431-015-5840-1.

3. Kakogawa, A.; Nishimura, T.; Ma, S. 2014. Designing arm length of a screw drive in-pipe robot for climbing vertically positioned bent pipes, Robotica 34(2): 306327.

https://doi.org/10.1017/S026357471400143X.

4. Zhang, Y.S.; Jiang, S.Y.; Zhang, X.W.; Yu, H.H.; Wang, D.L.; Guo, D.M. 2010. Dynamic characteristics of an intestine capsule robot with variable diameter, Chinese Science Bulletin 55(17): 1813-1821. https://doi.org/10.1007/s11434-009-3370-6.

5. Wang, Z.W.; Cao, Q.X.; Luan, N.; Zhang, L. 2010. Development of an autonomous in-pipe robot for offshore pipeline maintenance, Industrial Robot: An International Journal 37(2): 177-184. https://doi.org/10.1108/01439911011018957.

6. Kim, H. M., Choi, Y. S., Lee, Y. G., \& Choi, H. R. 2017. Novel mechanism for in-pipe robot based on a multiaxial differential gear mechanism, IEEE/ASME Transactions on Mechatronics 22(1), 227-235. https://doi.org/10.1109/TMECH.2016.2621978.
7. Tătar, O.; Mandru, D.; Ardelean, I. 2007. Development of mobile minirobots for in pipe inspection tasks, Mechanika 68(6):60-64.

8. Jeon, W. S.; Kim, I.; Park, J.W.; Yang, H. 2013. Design and control method for a high-mobility in-pipe robot with flexible links, Industrial Robot: An International Journal 40(3): 261-274. https://doi.org/10.1108/01439911311309960.

9. Adria, O.; Streich, H.; Hertzberg, J. 2004. Dynamic replanning in uncertain environments for a sewer inspection robot, International Journal of Advanced Robotic Systems 1(1): 33-38. https://doi.org/10.5772/5617.

10. Ma, Z.; Hu, Y.; Huang, J.; Zhang, X.; Wang, Y.; Chen, M.; Zhu, Q.M. 2007. A novel design of in pipe robot for inner surface inspection of large size pipes, Mechanics Based Design of Structures and Machines 35(4): 447-465. https://doi.org/10.1080/15397730701673296.

11. Li, P.; Ma, S.G.; Li, B.; Wang, Y. 2008. Multifunctional mobile units with a same platform for in-pipe inspection robots, IEEE/RSJ International Conference on Intelligent Robots and Systems, Nice, p. 22-26. https://doi.org/10.1109/IROS.2008.4650583.

12. Liu, Q.; Chen, Y.; Ren, T.; Wei, Y. 2014. Optimized inchworm motion planning for a novel in-pipe robot, Proceedings of the Institution of Mechanical Engineers Part C: Journal of Mechanical Engineering Science 228 (7):1248-1258. https://doi.org/10.1177/0954406213502409.

13. Ratansawanya, C.; Binsirawanich, P.; Yazdanjo, M.; Mehrandezh, M.; Poozesh, S.; Paranjape, R.; Najjaran, H. 2006. Design and development of a hardwarein-the loop simulation system for a submersible pipe inspecting robot, IEEE Canadian Conference on Electrical and Computer Engineering (CCECE), Ottawa, 15261529 . https://doi.org/10.1109/CCECE.2006.277638.

14. Xu, F.P.; Geng, C.; Xia, Y.S. 2012. Key techniques of robot for work of drainpipe deposit cleaning, Journal of Harbin Institute of Technology (New Series) 19(3): 9599 (in Chinese).

15. Kakogawa, A.; Ma, S.G. 2010. Mobility of an in-pipe robot with screw drive mechanism inside curved pipes, IEEE International Conference on Robotics and Biomimetics (ROBIO), Tianjin p. 1530-1535. https://doi.org/10.1109/ROBIO.2010.5723557.

16. Tang, D.W.; Li, Q.K.; Jiang, S.Y.; Deng, Z.Q.; Liu, H. 2011. Design and analysis of a pipeline robot with the function of differential movement, Journal of Mechanical Engineering 47(13): 1-8 (in Chinese). https://doi.org/10.3901/JME.2011.13.001.

17. Tatar, M.O.; Cirebea, C.; Mandru, D. 2012. The development of an in-pipe minirobot for various pipe sizes, IEEE International Conference on Automation Quality and Testing Robotics (AQTR), Cluj-Napoca, p. 443448. https://doi.org/10.1109/AQTR.2012.6237751.

18. Cai, Z.Q.; Lin, C.; Huo, D.H.; Zhu C.C. 2017. Design and analysis of cleaning mechanism for an intermittent screw-driven pipeline robot, Journal of Mechanical Science and Technology, 31(2): 911-921. https://doi.org/10.1007/s12206-017-0144-y. 
19. Hong, J.Z.; Liu, Z.Y.; Yang, C.J. 2015. Theoretical Mechanics, Higher Education Press, Beijing.

20. Li, Q.K.; Tang, D.W.; Jiang, S.Y.; Deng, Z.Q. 2012. Research and simulation on the driving property of a triaxial differential pipeline robot, Journal of Harbin Engineering University 33(6): 753-758 (in Chinese).

21. Tang, D.W.; Li, Q.K.; Jiang, S.Y.; Deng, Z.Q. 2010. Differential property and traction force of tri-axial differential pipeline robot in elbow, Robot, 32(1): 91-96 (in Chinese).

https://doi.org/10.3724/SP.J.1218.2010.00091.

22. Zhang C. 2008. Machinery Dynamics. Higher Education Press, Beijing.
G. Feng, W. Li, Z. Li, Z. He

\section{DEVELOPMENT OF A WHEELED AND WALL- PRESSING TYPE IN-PIPE ROBOT FOR WATER PIPELINES CLEANING AND ITS TRAVELING CAPABILITY}

\section{S u m m a r y}

Most of existing in-pipe robots are applied to defect inspection and regular maintenance, etc. There is almost no in-pipe robot for big-diameter pipelines cleaning and are still problems of traction force and obstacle-overcoming. To deal with the problems, a novel wheeled and wall-pressing type in-pipe cleaning robot (IPCR) with high pressure water jet is proposed. Then the preload mechanism, traction force, and obstacle-overcoming capability of the IPCR are analyzed. The vibration equations of the simplified vibration system of the IPCR are established. Also, the necessity of preloading force is verified and methods to improve obstacle-overcoming capability are proposed. Finally, the traveling capability is verified in a dynamics simulation system. The simulation results show that the proposed IPCR is powerful with traction force and has a certain capability of obstacle-overcoming. Setting the attitude angle is zero and adding preloading force to all springs can obviously weaken vibration of the IPCR.

Keywords: wheeled and wall-pressing type in-pipe cleaning robot (IPCR), preload mechanism, traction force, obstacle-overcoming capability, simplified vibration system.

Received August 08, 2017 Accepted April 15, 2020 